American Journal of Applied Sciences 5 (2): 136-141, 2008

ISSN 1546-9239

(C) 2008 Science Publications

\title{
Design of Low Phase Noise SIPC based Complementary LC-QVCO for IEEE 802.11a Application
}

\author{
Harikrishnan Ramiah and Tun Zainal Azni Zulkifli \\ RFIC Design Group, Universiti Sains Malaysia, Engineering Campus \\ 14300 Nibong Tebal, Seberang Perai Selatan, Penang, Malaysia
}

\begin{abstract}
The paper presents the design of a source injection parallel coupled (SIPC) quadrature voltage controlled oscillator (QVCO), realized in a complementary architecture, which is usually preferred in low-power applications as it exploits $\approx 50 \%$ bias current reduction with double efficiency compared to the structure with single coupled, when operating in the current-limited regime. A stacked spiral inductor exhibiting a Q factor of 5.8, with pMOS based depletion mode varactor of $32 \%$ in tuning range, corresponding to $3.2-3.6 \mathrm{GHz}$ of tuning frequency, is implemented in $0.18 \mu \mathrm{m} \mathrm{CMOS}$ technology. The phase noise of the SIPC QVCO architecture simulated at $1 \mathrm{MHz}$ of offset frequency is indicated to be $-114.3 \mathrm{dBc} / \mathrm{Hz}$, while dissipating $11.0 \mathrm{~mW}$ of core circuit power.
\end{abstract}

Key words: $\quad$ Phase noise, pMOS varactor, Quadrature voltage controlled oscillator (QVCO), Source injection parallel coupled VCO (SIPC-QVCO), Stacked spiral inductor

\section{INTRODUCTION}

Increasing demand for higher capacity in the growing LAN (WLAN) market has led to the introduction of a new generation of WLAN standards using more spectrally efficient modulation techniques. The 802.11a standards are based on orthogonal frequency division multiplexing (OFDM) modulation, where 52 uniformly spaced carriers are independently modulated with PSK or QAM, hence requires circuit architecture with low amplitude, high linearity and low phase mismatch, thus improved Error Vector Magnitude (EVM) performance. The standard supports data rates from 6 to $54 \mathrm{Mbps}$ in the $5 \mathrm{GHz}$ unlicensed national information infrastructure (UNII) band (5.15$5.35 \mathrm{GHz})[1]$.

In the transmitter the PA (Power Amplifier) output contains large spectral components in the vicinity of $f_{L O}$, leaking through the package and the substrate to the VCO and causing pulling. This effect is known as injection pulling [2]. To alleviate this effect proper frequency planning is utilized in the two-step upconversion mixing action of the RF transmitter [3].
The accuracy in reported QVCO architecture designed utilizing a RC-CR polyphase filter is strongly dependent on the on-chip component matching, whereas the usage of frequency divider in realizing the QVCO topology increases the power consumption and results in quadrature inaccuracy due to any asymmetry in the duty cycle of the master-slave D-flip flop based frequency divider [4]. A cross coupled QVCO topology is exploited to enhance the phase accuracy. In this paper a SIPC based QVCO topology is adapted [5]. By alleviating the noise current flow through the switching transistor from the coupling transistor the up conversion of the $1 / f$, flicker noise is relaxed.

A stacked spiral inductor with comparable $Q$ factor and self resonant frequency is designed [6] and integrated into the QVCO architecture to provide frequency tuning via the LC tank configuration. A metal6-metal4 stacked rectangular spiral inductor which provides self shielding effect is implemented and extracted utilizing ASITIC (Analysis and Simulation of Inductors and Transformer for IC's) tool.

A pMOS based capacitor with drain, source and bulk (D,S,B) connected together [7], realizes the frequency tuning of the LC tank. Both in accumulation

Corresponding Author: Tun Zainal Azni Zulkifli, RFIC Design Group, School of Electrical \& Electronic Engineering, Universiti Sains Malaysia, 14300 Nibong Tebal , Penang, Malaysia, Tel: +604-5996010, Fax: $+604-5941023$ 
and inversion region of operation the MOS capacitor exhibit a maximum of oxide capacitance, $C_{o x}$.

This paper is organized as follows. The frequency planning scheme description for IEEE 802.11a application is followed by the review on the design of the stacked spiral inductor, subsequently by the utilized pMOS based capacitor and the description of the integrated complementary based SIPC-QVCO architecture. Simulation results on the realized SIPC based QVCO in $0.18 \mu \mathrm{m}$ CMOS technology is reported prior to the presented conclusion.

\section{FREQUENCY PLANNING}

Figure 1 reports an LO generation scheme utilized in realizing two step up-conversion transmitter in compliance with IEEE 802.11a application. The voltage controlled oscillator (VCO) operates at two thirds of the LO frequency and a divide by 2 circuit produces quadrature outputs at one third of LO frequency. As the VCO operates at two thirds of the LO frequency, this scheme effectively suppresses the injection pulling effect and LO-RF interaction by subsequent stage of PA. The generated LO signal has a cleaner frequency content at the LO frequency, minimizing the adverse effect of the unwanted sideband [8].

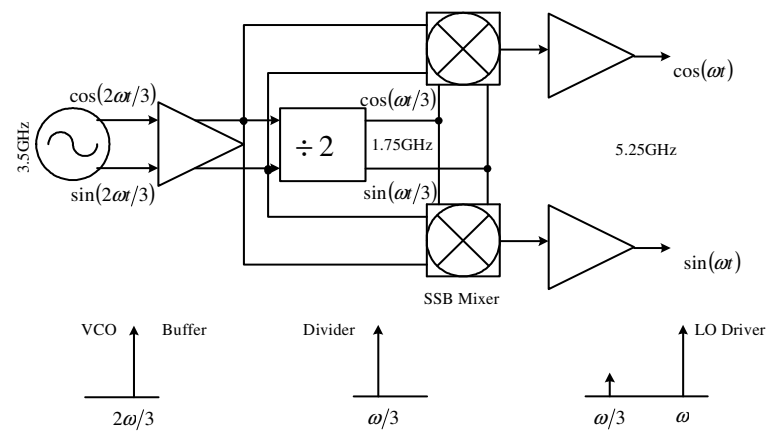

Fig. 1: Proposed frequency planning for IEEE 802.11a Transmitter architecture [6]

\section{ARCHITECTURE AND CIRCUIT IMPLEMENTATION}

Stacked Spiral Inductor: Figure 2 illustrates the vertical cross section of the realized stacked inductor, with the indication of substrate and oxide thickness. Figure 3(a) illustrates the layout view of the designed inductor with patterned grounded shield (PGS), which terminates the induced electric field and prevents it from reaching the substrate. Figure 3(b) describes the equivalent circuit model with PGS [6],

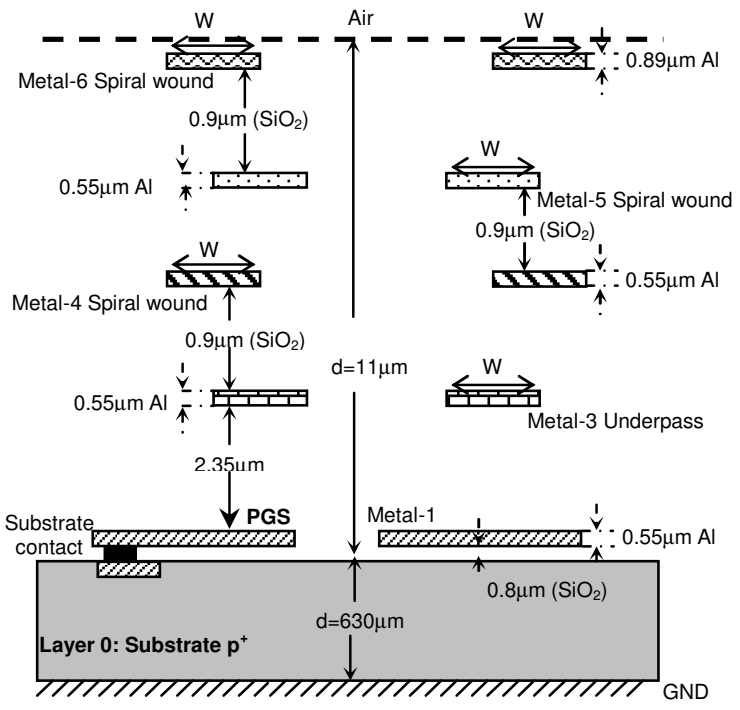

Fig 2: $\quad$ Spiral inductor cross section showing various layers metal 6 to metal 4 is used for creating the turns of the spiral with an underpass layer of metal 3 . The PGS is designed in metal 1.

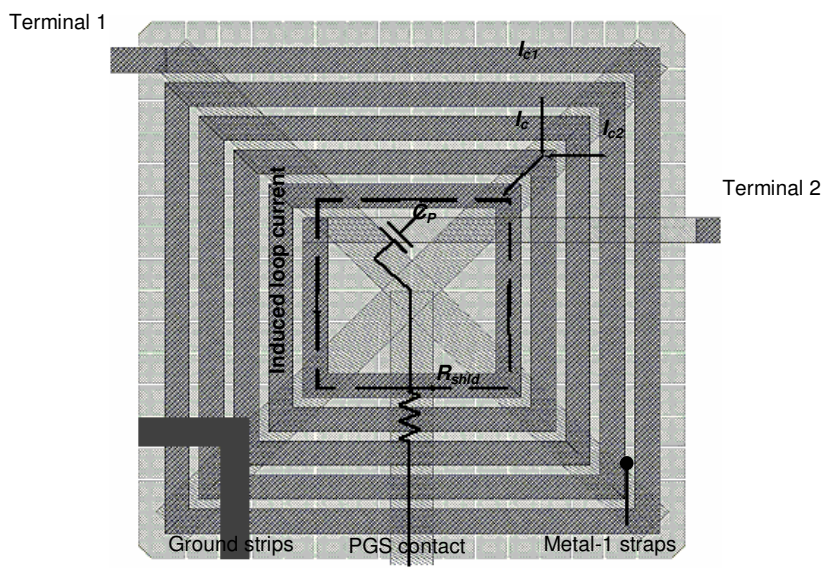

(a)

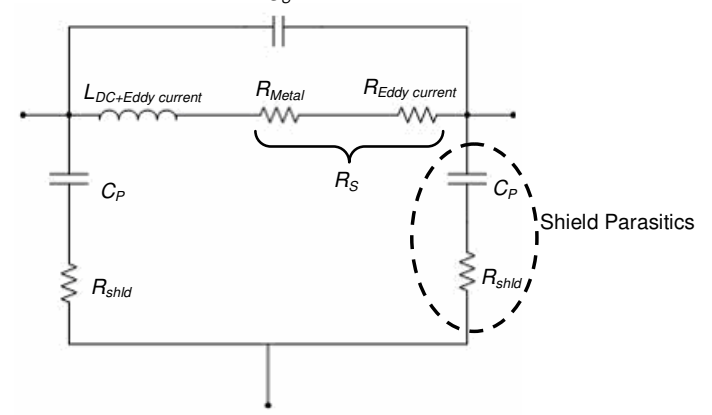

(b)

Fig. 3: $\quad$ Stacked spiral inductor (a) layout view with PGS, metal 1 straps and substrate parasitic distribution and (b) equivalent circuit model. 
where $L_{D C+E d d y \text { current }}$, is the equivalent inductance due to the dc self inductance and mutual coupling inductance, $R_{\text {Metal }}$ and $R_{\text {Eddy current, denotes the dc metal line and }}$ reverse eddy current induction losses, respectively, $C_{S}$ is the series capacitance due to the effect of interwinding and overlapping capacitance effect, whereas $C_{p}$ and $R_{\text {shld }}$ are the shield parasitics.

\section{pMOS Varactor}

The gate of the device is defined as anode $v^{+}$, while the source, drain and bulk are tied together, forming the cathode, $v^{-}$, of the device as described in Figure 4. The varactor gate sided tuning is used to alleviate leakage.

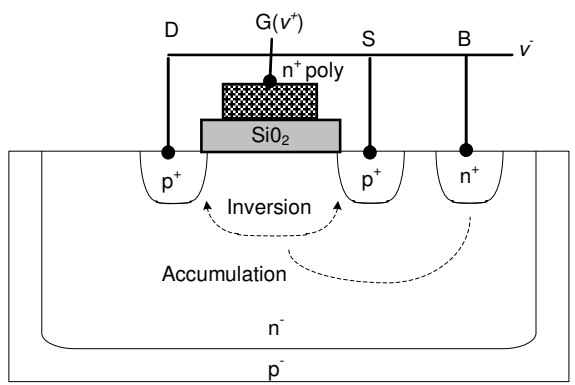

Fig. 4: pMOS cross section describing MOS capacitor in inversion, depletion and accumulation regions.

Inversion channel with mobile holes builds up for $V_{B G}>\left|V_{T}\right|$, where $\left|V_{T}\right|$ is the threshold voltage of the transistor, resulting in an equivalent capacitance, $C_{o x}$ [7]:

$$
C_{o x}=\frac{\varepsilon_{o x} A}{t_{o x}}
$$

where $A$ and $t_{o x}$ are the transistor channel area and oxide thickness, respectively.

The depletion mode with very few mobile charge carriers, results in a decrease of the equivalent capacitance to $C_{o x}+C_{d}$, where $C_{d}$ is the series depletion mode capacitance. The varactor $Q$ is defined by [7]:

$$
Q=\frac{1}{\omega C_{V} R_{V}}
$$

where $R_{V}$ is the series parasitic resistance including the channel resistance and gate resistance, which is reduced utilizing minimal length double sided tapped gate MOS varactor with multi finger realization. $C_{V}$ describes the varactor capacitance, where $C_{V}=C_{o x}$.W.L.nf, in which $W, L$ and $n f$ corresponds to the width $(\mu \mathrm{m})$, length $(\mu \mathrm{m})$ and number of fingers of the varactor, respectively.

\section{Complementary Based SIPC-QVCO}

Figure 5 shows the schematic of the complementary based SIPC-QVCO topology. In contrary to the conventional QVCO architecture, the drain node $A$ of the coupling transistor, $M_{C l}-M_{C 4}$ are connected directly to the ground (GND) terminal.

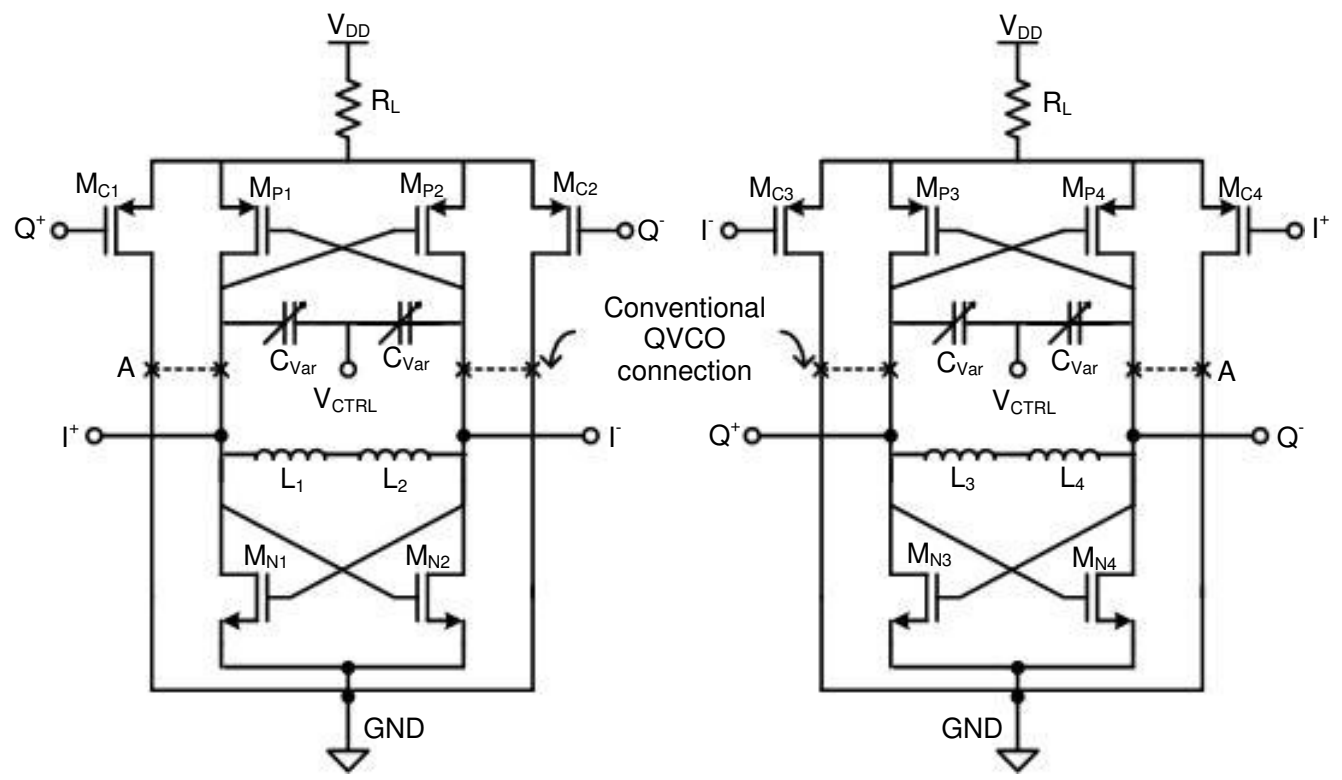

Fig. 5: Complementary stage SIPC-QVCO 
Phase noise in the $1 / f^{3}$ region is primarily dependant upon the up-conversion of flicker noise from the MOS switching transistors, $M_{N I}-M_{N 4}$. Bias current noise has been observed to be a dominant contributor to phase noise in the $1 / f^{2}$ region, solutions are filtering and removing the bias current generator where filtering requires large inductors and capacitors, while removing current source increases sensitivity to power supply noise and variation of the bias current over process and temperature, hence the utilization of common mode resistor to the biasing supply voltage is preferred. There is no particular reason in choosing an nMOS or a pMOS device with regard to $1 / f^{2}$ phase noise in selecting the coupling transistor pair, largely because the device have been assumed to yield equivalent thermal noise for an equivalent $g_{m}$. The reduction in $1 / f^{3}$ phase noise is fundamentally limited by switching transistors $1 / f$ noise [9].

In a conventional QVCO architecture, the resulting 1/ $f$ noise current flows through the switching transistor $M_{N I}-M_{N 4}$. When the coupling transistors turns off, the abrupt change in noise current leads to a voltage induction across $M_{N I}-M_{N 4}$, which up-converts the $1 / f$ noise to the frequency of oscillation, thus degrading the phase noise performance [5]. The SIPC-QVCO architecture contributes no voltages induction across $M_{N 1}-M_{N 4}$ since the drain node of $M_{C l}-M_{C 4}$ are directly connected to GND, thus relaxing the $1 / f$ upconversion.

The quadrature generation is obtained through coupling of two identical oscillators in such a way that forces their outputs to oscillate $90^{\circ}$ out of phase, with a tradeoff of two times in area and power utilization of a single LC oscillator [4].

\section{SIMULATION RESULTS}

Figure 6 illustrates the inductance and quality factor variation of the designed stacked spiral inductor extracted utilizing ASITIC tool. The designed inductor consumes an area of $100 \times 100 \mu \mathrm{m}^{2}$ with 3.25 number of spiral turns. From Figure 6 it is evident that the inductance variation is somewhat constant with frequency. At frequency well below the peak in the inductor $Q$, the shunt parasitic of the spiral inductor has little effect and consequently the inductive reactance and $Q$ factor increases with frequency. However as the operating frequency continues to rise, the energy dissipation in the semiconducting substrate and the ac resistance of the metallization begins to increase faster than the inductive reactance, thus the $Q$ factor peaks and then decreases.

Figure 7 describes the tuning characteristic of the designed pMOS based capacitor. From Figure 7, when the diffusion voltage is constant and the dc voltage applied at the gate $V_{C T R L}$, is increased the MOS capacitor varies from the inversion region to the accumulation region of operation. The simulated varactor consists of 130 segments with a total gate dimension of $650 \mu \mathrm{m} \times 0.18 \mu \mathrm{m}$. The size of each segment is $5 \mu \mathrm{m} \times 0.18 \mu \mathrm{m}$.

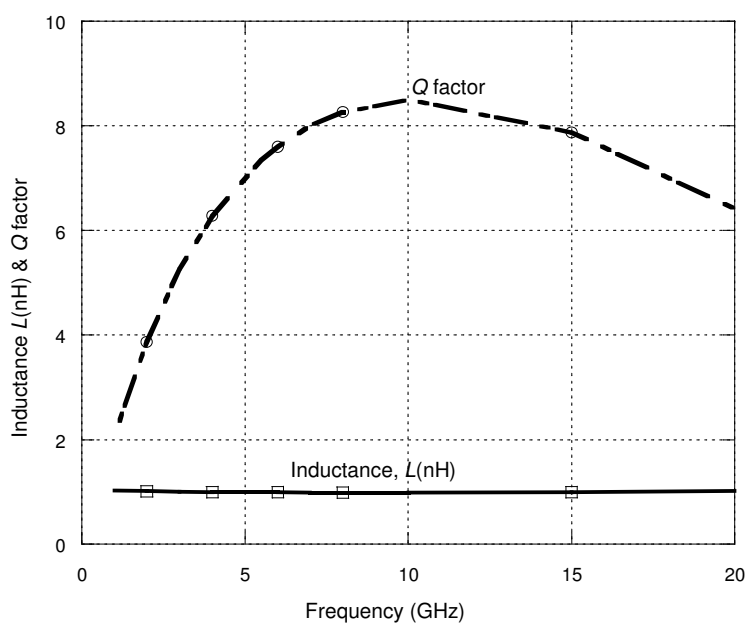

Fig. 6: Inductance $\mathrm{L}$ and $\mathrm{Q}$ factor characteristic

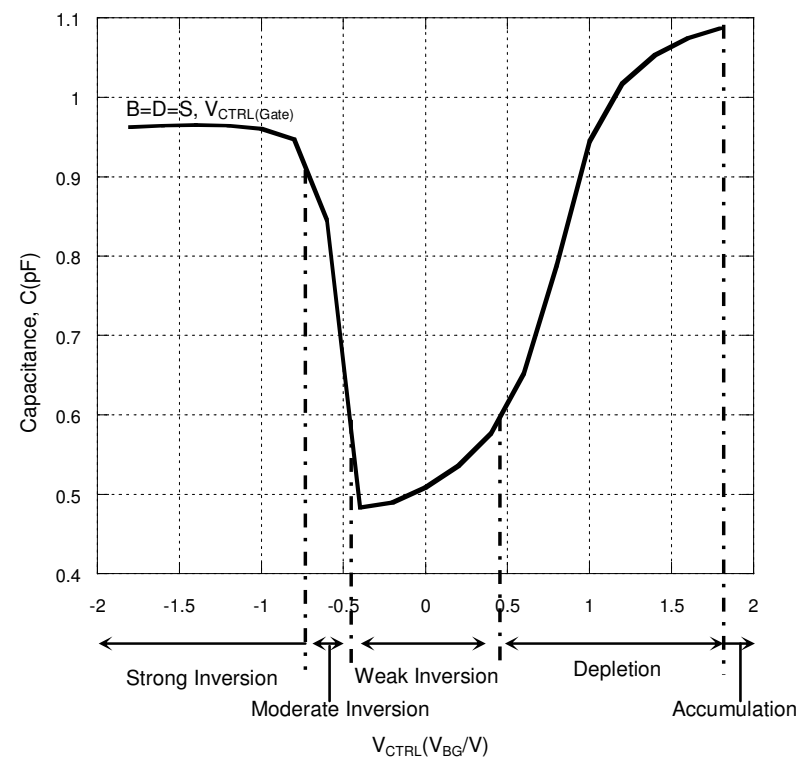

Fig. 7: Tuning characteristic for pMOS based capacitor with $\mathrm{B}=\mathrm{D}=\mathrm{S}$ 
Figure 8 describes the pre-layout phase noise performance of the designed pMOS based SIPC-QVCO in comparison with conventional LC-QVCO topology. It is observed that the SIPC-QVCO has superior close in phase noise suppression. Figure 9 describes the prelayout tuning characteristic of the designed QVCO. The tuning frequency ranges from approximately 3.23.6GHz.

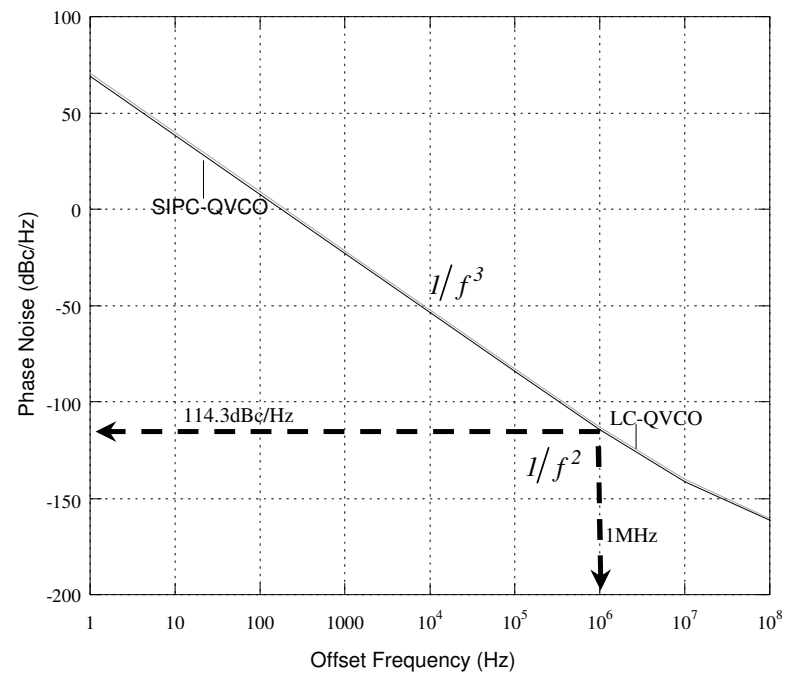

Fig. 8: Simulated phase noise versus offset frequency at 3.5GHz.

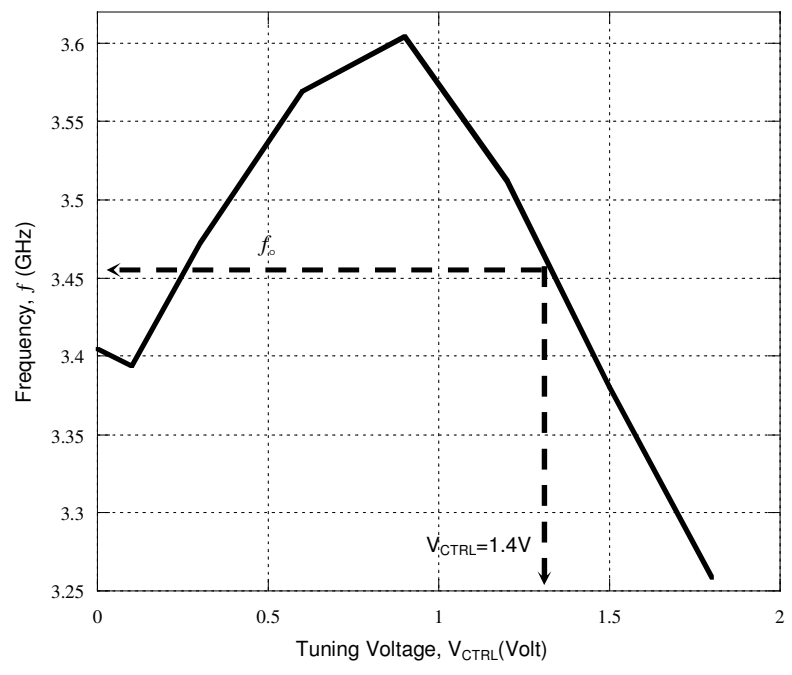

Fig. 9: Tuning characteristic of the complementary SIPC-QVCO

Figure 10 illustrates the physical implementation of the complementary SIPC- QVCO, implemented in $0.18 \mu \mathrm{m}$ CMOS technology and consumes a dimension of $1.9 \times 1.16 \mathrm{~mm}^{2}$. The layout focuses on the symmetry of the balanced circuit.

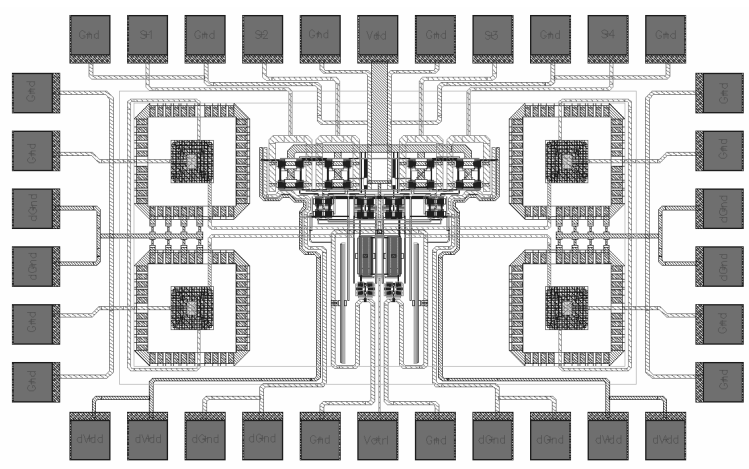

Fig. 10: Complementary SIPC-QVCO physical layout

Table I summarizes the simulated performance of the fully integrated complementary SIPC-QVCO.

\begin{tabular}{ll} 
Table I: Complementary SIPC-QVCO performance summary \\
\hline Parameter & Result \\
\hline \hline Supply Voltage & $1.8-\mathrm{V}$ \\
Tuning Range & $400-\mathrm{MHz}$ \\
Tuning Voltage & $0.9 \sim 1.8-\mathrm{V}$ \\
$f$ & $3.45-\mathrm{GHz}$ \\
Po & $-114.3 \mathrm{dBc} / \mathrm{Hz} @ 1 \mathrm{MHz}$ \\
Phase Noise & \\
Power Dissipation & $11 \mathrm{~mW}$ \\
QVCO Core & $115 \mathrm{~mW}$ \\
Open Drain Buffer &
\end{tabular}

\section{CONCLUSION}

A low phase noise differential complementary based SIPC QVCO in comparison of the conventional LC-QVCO architecture is presented in compliance with IEEE 802.11a two step up-conversion outlines. An investigation of the stacked spiral inductor and pMOS varactor is performed in optimizing the design of the realized QVCO architecture.

\section{ACKNOWLEDGMENT}

The authors gratefully acknowledge Albert Victor Kordesch from Silterra Malaysia Sdn.Bhd., for the fabrication of the experimental ICs in $0.18 \mu \mathrm{m}$ CMOS technology.

\section{REFERENCES}

1. B. Benny, 2002. Wireless Local Area Networks: The New Wireless Revolution. New York: John Wiley \& Sons, Inc., pp: 73-83. 
2. B. Razavi, 1998. RF Microelectronics. United States: Prentice Hall PTR, pp:152-155.

3. Z. Masoud, K.S. David, Y. Patrick, R. Shahriar, W. David, J. K. Brian, S. M. Srenik, S. Kalwant, M. Sunetra and A. W. Bruce, 2002. A 5-GHz CMOS Transceiver for IEEE 802.11a Wireless LAN Systems. IEEE J.Solid-State Circuit., 37: 16881694.

4. R.Aparicio, and A.Hajimiri, 2002. A NoiseShifting Differential Colpitts VCO. IEEE J. SolidState Circuits., 37(12): 1728-1736.

5. H.C.Choi, S.B.Shin, and S.G.Lee, 2004. A LowPhase Noise LC-QVCO in CMOS Technology. IEEE Microwave and Wireless Comp. Lett., 14(11): 540-542.
6. N.Talwalkar, 2003. Integrated CMOS Transmit Receive Switch using On-Chip Spiral Inductor. Ph.D dissertation, Dept. Elect. Eng., Stanford University, California.

7. P.Andreani, and S.Mattison, 2000. On the Use of MOS Varactors in RF VCO's. IEEE J. Solid-State Circuits., 35(6): 905-910.

8. Z. Pengfei, N. Thai, L. Christopher, G. Douglas, S. Theerachet, C. Baohing, H. Siegfried, S. Isaac, B. Taofik, T. Andrew and B. Razavi, 2003. A 5-GHz Direct-Conversion CMOS Transceiver. IEEE J.Solid-State Circuit., 38: 2232-2238.

9. A.Jering, C.G.Sodini, 2005. The Impact of Device Type and Sizing on Phase Noise Mechanisms. IEEE J. Solid-State Circuits., 40: 360-369. 